Published in final edited form as:

Gynecol Oncol. 2017 August ; 146(2): 234-239. doi:10.1016/j.ygyno.2017.05.016.

\title{
A prospective validation study of sentinel lymph node mapping for high-risk endometrial cancer
}

\author{
Pamela T. Soliman, MD ${ }^{1,}{ }^{*}$, Shannon N. Westin, MD ${ }^{1}$, Shayan Dioun, MD ${ }^{1}$, Charlotte C. Sun ${ }^{1}$, \\ Elizabeth Euscher, MD ${ }^{2}$, Mark F. Munsell ${ }^{3}$, Nicole D. Fleming, MD ${ }^{1}$, Charles Levenback, \\ MD ${ }^{1}$, Michael Frumovitz, MD¹, Pedro T. Ramirez, MD¹, and Karen H. Lu, MD ${ }^{1}$ \\ ${ }^{1}$ Department of Gynecologic Oncology and Reproductive Medicine, University of Texas, M.D. \\ Anderson Cancer Center, Houston, Texas \\ ${ }^{2}$ Department of Pathology, University of Texas, M.D. Anderson Cancer Center, Houston, Texas \\ ${ }^{3}$ Division of Biostatistics, University of Texas, M.D. Anderson Cancer Center, Houston, Texas
}

\section{Abstract}

Objective-Sentinel lymph node (SLN) mapping continues to evolve in the surgical staging of endometrial cancer (EC). The purpose of this trial was to identify the sensitivity, false negative rate (FNR) and FN predictive value (FNPV) of SLN compared to complete pelvic and para-aortic lymphadenectomy (LAD) in women with high-risk EC.

Methods-Women with high-risk EC (grade 3, serous, clear cell, carcinosarcoma) were enrolled in this prospective surgical trial. All patients underwent preoperative PET/CT and intraoperative SLN biopsy followed by LAD. Patients with peritoneal disease on imaging or at the time of surgery were excluded. Patients were evaluable if SLN was attempted and complete LAD was performed.

\begin{abstract}
Results-123 patients were enrolled between 4/13 and 5/16; 101 were evaluable. At least 1 SLN was identified in $89 \%$ (90); bilateral detection $58 \%$, unilateral pelvic $40 \%$, para-aortic only $2 \%$. Indocyanine green was used in $61 \%$, blue dye in $28 \%$, and blue dye and technetium in $11 \%$. Twenty-three pts (23\%) had $\geq 1$ positive node. In 20/23, $\geq 1$ SLN was identified and in 19/20 the SLN was positive. Only 1 patient had bilateral negative SLN and positive non-SLNs on final pathology. Overall, sensitivity of SLN was 95\% (19/20), FNR was 5\% (1/20) and FNPV was 1.4\% (1/71). If side-specific LAD was performed when a SLN was not detected, the FNR decreased to $4.3 \%(1 / 23)$.
\end{abstract}

\footnotetext{
* To whom correspondence and reprint requests should be addressed at Department of Gynecologic Oncology and Reproductive Medicine, CPB6.3244, Unit 1362, The University of Texas MD Anderson Cancer Center, 1155 Herman Pressler, TX 77030. Phone: (713) 745-2352. Fax: (713) 792-7586. psoliman@mdanderson.org.

This work was presented as an oral abstract at the Society of Gynecologic Oncology Annual Meeting in 2015 in Los Angeles, California and at the International Gynecologic Cancer Society Annual Meeting in 2016 in Lisbon, Portugal.

Declaration of interests

Michael Frumoviz (Novadaq research funds and consultant, Navidea research funds). All other authors have no conflicts to disclose.

Publisher's Disclaimer: This is a PDF file of an unedited manuscript that has been accepted for publication. As a service to our customers we are providing this early version of the manuscript. The manuscript will undergo copyediting, typesetting, and review of the resulting proof before it is published in its final citable form. Please note that during the production process errors may be discovered which could affect the content, and all legal disclaimers that apply to the journal pertain.
} 
Conclusion-This prospective trial demonstrated that SLN biopsy plus side-specific LAD, when SLN is not detected, is a reasonable alternative to a complete LAD in high-risk endometrial cancer.

\section{Introduction}

Endometrial cancer is the most common gynecologic cancer in the United States with 60,050 new cases estimated for 2016 (1). There continues to be significant controversy on the surgical management of these patients. Two randomized studies evaluating the role of lymphadenectomy in endometrial cancer showed no benefit in disease-free or overall survival $(2,3)$. Despite these findings, many agree that the identification of metastatic disease in the lymph nodes is critical in the diagnosis and treatment of women with endometrial cancer and lymph node metastases are an important prognostic factor in overall survival (4) (5). The use of sentinel lymph node (SLN) mapping plus ultra-staging could potentially maximize the identification of positive nodes, while minimizing the known risks of lymphadenectomy including longer surgical times, intraoperative injury, blood loss, and lymph edema $(6,7)$.

There is a growing body of evidence supporting the role of SLN mapping in endometrial cancer. Published studies have described SLN detection rates as high as $85 \%$ to $100 \%$ with bilateral detection rates of $60 \%$ to $97 \%$ (8-13). Memorial Sloan Kettering developed a SLN algorithm that includes performing a side-specific pelvic lymphadenectomy when a SLN is not detected (14). Retrospective implementation of this algorithm resulted in a significant decrease in their false negative rate from $15 \%$ to $2 \%$ in women with low-risk endometrial cancer. Based on this and other studies, SLN mapping has been recognized as an option for nodal assessment in the 2014 National Comprehensive Cancer Network (NCCN) guidelines for endometrial cancer (15).

The majority of studies, however, include patients at low-risk for lymph node involvement and may underestimate the false negative rate. In order to consider SLN biopsy alone in women with high-risk endometrial cancer, a prospective validation study requiring comprehensive surgical staging was needed.

The primary objective was to estimate the false negative rate (FNR) of SLN mapping in the detection of positive lymph nodes in women with high-risk endometrial cancer. We hypothesized that SLN mapping would accurately identify women with positive lymph nodes with an acceptable false negative rate.

\section{Methods}

After institutional board review approval, patients were prospectively enrolled in this single arm study at MD Anderson Cancer (NCT01737619). All patients who presented with newly diagnosed endometrial cancer were consecutively screened for eligibility. Patients were eligible if they had high-risk endometrial cancer including serous, clear cell, FIGO grade 3 endometrioid, or carcinosarcoma histology based on preoperative sampling. Patients with biopsy-proven cervical involvement or FIGO grade 1/2 endometrioid tumors with suspected deep myometrial invasion on imaging were also eligible. Patients had to be a candidate for 
full surgical staging. Once written informed consent was confirmed, patients underwent preoperative PET/CT. Patients with suspected peritoneal disease were excluded from the study.

All patients were offered minimally invasive surgery unless they were not felt to be a candidate. The decision to perform surgery robotically versus traditional laparoscopy was based on surgeon preference, as both are considered standard of care. The type of dye used and the timing of the injection for the mapping procedure was based on the surgical approach (Table 1).

At the time of surgery, all patients underwent intraoperative SLN mapping with a cervical injection of dye in equally divided aliquots both superficial (submucosal) and deep (1 cm into the stroma), at 3 and 9 o' clock. Sentinel nodes were removed, labelled (blue, green or hot including gamma count) and sent to pathology for permanent section. Full surgical staging was performed with the goal of removing para-aortic nodes to the level of the renal vessels. If peritoneal disease was identified intra-operatively, patients were no longer eligible.

The primary outcome was to estimate the false negative rate (FNR) of SLN mapping in the detection of positive lymph nodes in women with high-risk endometrial cancer. We also estimated the sensitivity, negative predictive value (NPV) and false negative predictive value (FNPV) when compared to final pathology. A sample size of 100 patients was identified based on an estimated $25 \%$ node positivity rate. Patients were evaluable if SLN mapping was attempted (i.e. dye was injected) and a full lymphadenectomy was performed. We employed a Bayesian monitoring rule using the method of Thall et al. to guard against a FNR of more than $10 \%$ (90\% CI $1.4 \%$ to $13.8 \%$ ) (16). We assumed we would have 25 patients with positive nodes among the 100 evaluable patients, and we applied our monitoring rule after every cohort of 5 evaluable patients. We assumed a uniform prior distribution for the FNR. If, given the outcomes from the evaluable patients, there was more than a $95 \%$ chance of a FNR more than $10 \%$ we would have stopped the study.

All pathologic specimens were reviewed by a gynecologic pathologist. The SLNs were processed and classified according to a modification of the AJCC staging for axillary nodes from breast cancer. If the $\mathrm{SLN}$ was $<5 \mathrm{~mm}$, it was bi-valved. If $\geq 5 \mathrm{~mm}$ it was serially sectioned every $2 \mathrm{~mm}$. An $\mathrm{H} \& \mathrm{E}$ was performed on each section. If negative, an additional wide H\&E stained slide plus 2 unstained slides were obtained $250 \mu \mathrm{m}$ into the tissue block. A pan-cytokeratin stain was performed when the deeper H\&E level was negative. SLNs were classified as 1) metastases present - tumor $>2.0 \mathrm{~mm}$; 2) micrometastases - tumor cell aggregates between 0.2 and $2.0 \mathrm{~mm} ; 3$ ) isolated tumor cells - individual tumor cells or aggregates that are $<0.2 \mathrm{~mm}$, or 4) negative. If the pan-cytokeratin stain was positive but no tumor cells were identified, this was considered negative for metastatic disease. Non-sentinel lymph nodes were reported as positive or negative for metastases based upon routine sectioning and examination of a single H\&E stained slide per standard protocol.

All lymph nodes with macroscopic, microscopic and isolated tumor cells were considered to be positive on final pathology. In defining a false negative we looked at both the patient 
(primary objective), as well as the technique by hemi-pelvis. A patient was considered a false negative if they had bilateral SLN biopsies that were negative and a non- sentinel lymph node that was positive. When we assessed the technique by hemi-pelvis, a hemipelvis was considered a false negative if there was a SLN biopsy that was negative and a non-sentinel lymph node on the same side of the pelvis that was positive.

We used descriptive statistics to summarize the demographic and clinical characteristics of patients. We estimated sensitivity, NPV, FNR, and FNPV with 95\% exact binomial confidence intervals. All statistical analyses were performed using SAS 9.3 for Windows (Copyright $\odot$ 2002-2010 by SAS Institute Inc., Cary, NC) and StatXact-7@ for Windows (Copyright @ 2005, 1989-2005, Cytel Software Corporation, Cambridge, Massachusetts). Study data were collected and managed using REDCap (Research Electronic Data Capture) electronic data capture tools hosted at MD Anderson (17). REDCap is a secure, web-based application designed to support data capture for research studies, providing: 1) an intuitive interface for validated data entry; 2) audit trails for tracking data manipulation and export procedures; 3 ) automated export procedures for seamless data downloads to common statistical packages; and 4) procedures for importing data from external sources.

\section{Results}

Between April 2013 and May 2016, a total of 123 patients were enrolled (Figure 1). Twentytwo patients were excluded for the following reasons: non-surgical management of their disease (8), SLN mapping not performed (12) or lymphadenectomy not performed (3). The remaining 101 were considered evaluable for the final analysis.

The demographic characteristics of the evaluable patients are shown in Table 2. Median age was 62 years (range 29 - 86). Median body mass index (BMI) was $30.8 \mathrm{~kg} / \mathrm{m}^{2}$ (range $15.8-$ 64.3). Endometrioid (44\%) and serous (30\%) were the most common histologies. A majority of cases (84\%) were done minimally invasive. On final pathology, $73 \%$ had early-stage and $27 \%$ had advanced disease.

The overall SLN detection rates are shown in Table 3. ICG was the most commonly used dye $(\mathrm{n}=62,61 \%)$. The overall SLN detection rate was $89 \% ; 58 \%$ bilateral pelvic, $40 \%$ unilateral pelvic, and $2(2 \%)$ para-aortic. While bilateral detection rate appeared to be highest with blue dye + technetium, the study was not powered to detect a difference in rates between techniques $(p=0 \cdot 35)$. There were no reported adverse events directly related to the SLN mapping.

The median number of pelvic nodes per patient was 17 (range 4-36) and the median number of para-aortic nodes was 6 (range 0-28). The upper border of the para-aortic dissection was the renal vessels in 47 patients (46.5\%), above the inferior mesenteric artery (IMA) but the renal vein not visualized in $29(28.7 \%)$, to the IMA in $18(17.8 \%)$, and below the IMA in 2 (2\%). Para-aortic lymphadenectomy was not performed in 5 due to difficult visualization.

Among the 90 patients (89\%) in whom $\geq 1$ SLN was detected, the median number was 2 (range 1-9) per patient. Two hundred twenty-seven nodes were labeled SLN. Twelve (5.2\%) were in the para-aortic region. Two hundred fourteen (94.2\%) were pelvic; 50.2\% external 
iliac, $39.8 \%$ obturator, $4.8 \%$ common iliac, and one parametrial sentinel node. There were a total of 31 positive SLN among 19 patients; 21 were external iliac and 10 were obturator nodes. The sentinel nodes were categorized by size including $3 / 28(11 \%)$ isolated tumor cells, 9/28 (32\%) micrometastasis, 16/28 (57\%) macrometastasis, with 3 unknown.

There were 23 node positive patients based on final pathology. Among these, 3/23 (13\%) did not have a SLN detected at the time of surgery. Among the 20 who had $\geq 1$ SLN identified, 19/20 (95\%) had at least 1 positive SLN. In 8/20 (40\%), the SLN was the only positive node. Fifty-two percent (12/23) had $\geq 1$ positive non-sentinel pelvic lymph node (median 1; range $0-9)$. Forty-eight percent (11/23) had positive para-aortic nodes. One patient had bilateral negative SLNs and a positive non-SLN, therefore, considered a true false negative. One patient had isolated positive para-aortic nodes but did not have any sentinel nodes detected.

The $2 \times 2$ tables evaluating the efficacy of SLN biopsy alone are shown in Table 4 . When evaluating the technique by hemi-pelvis, there were 126 pelvic basins for which $\geq 1$ SLN was detected. The sensitivity to detect a positive node in the hemi-pelvis was $92.9 \%$, FNR $7.1 \%$, NPV $98 \%$ and FNPV $2 \%$. When evaluating the efficacy of SLN biopsy per patient (Table 4B): the sensitivity was $95 \%$, FNR 5\%, NPV 98.6\% and FNPV 1.4\%. When including side-specific lymphadenectomy when a SLN was not identified, the FNR was $4.3 \%(1 / 23)$.

\section{Discussion}

In this prospective validation study of SLN mapping in women with high-risk endometrial cancer, SLN biopsy alone accurately identified $95 \%$ of patients with positive lymph nodes. When combined with side-specific lymph node dissection, the FNR was 4.3\%. The FNPV, i.e. the likelihood of a missed positive node if the SLN was negative, was 1.4\%. These findings support SLN mapping in high-risk endometrial cancer and validate the algorithm of side-specific lymphadenectomy if a SLN is not identified.

The original studies evaluating SLN for endometrial cancer included both fundal and hysteroscopic injection of dye into the tumor $(18,19)$. Fundal injection has been associated with detection rates around 45\% (20). More promising data has come from hysteroscopic peri-tumoral injection, however, the ease and feasibility of this technique has limited its adoption by many centers, including our own (19). Based on these factors, we chose to use a cervical injection of dye; both superficial and deep at 3 and 9 o'clock as previously reported by early adopters of SLN in endometrial cancer (21). At the initiation of this study in early 2013, there were little data comparing detection rates based on dye type, therefore the dye used was based on the surgical approach and available resources. For example, a laparoscopic gamma probe was not available at one of our satellite hospitals (LBJ) and equipment needed for SLN mapping with ICG during laparoscopy was not available at our institution until November, 2015.

Our overall SLN detection rate was $89 \%$, with a bilateral detection rate of $58 \%$. This was consistent with a recent meta-analysis by Smith et al. which reported an overall detection rate of $81 \%$ and a bilateral detection rate of $50 \%$ when compiling data from 55 published 
studies on endometrial cancer SLN mapping (22). While our study was not powered to detect a difference in detection rates by mapping technique, several studies have suggested that the use of ICG is associated with higher overall and bilateral detection rates compared to blue dye (9). Recent studies have reported overall detection rates of $95-97 \%$, with bilateral detection rates of 79-88\% (9-11). This study was initiated during the early learning curve of sentinel lymph node mapping for our institution and this likely had an impact on our mapping success. Although there are no published data looking specifically at the learning curve for SLN in endometrial cancer, we anticipate that with advancements in technology and further experience, the bilateral detection rates for all surgeons will improve.

In our study, SLN biopsy alone identified $95 \%$ of node positive women, resulting in a FNR of 5\%. These are higher than the SENTI-ENDO study which reported a sensitivity of $84 \%$ in women with any grade endometrial cancer (13). A pooled analysis done by Smith et al. reported a sensitivity of $96 \%$ and a NPV of $99.7 \%$ (22). Barlin et al. first described the implementation of a SLN algorithm that resulted in a significant decrease in their false negative rate (14). If one includes a side-specific lymphadenectomy when a SLN was not detected, our FNR decreased from 5 to $4.3 \%$. While implementation of the algorithm improves overall accuracy of SLN mapping, it also allows the surgeon to go through the natural learning curve without compromising the care of the patient. For example, if a surgeon's SLN detection rate is low, performance of the side-specific lymphadenectomy would ensure they did not miss a positive node on a side that did not map. As surgeon experience improves, the need for performing a lymphadenectomy would decrease as bilateral detection rates increased.

In vulvar cancer, after several single institution studies demonstrated feasibility of SLN biopsy $(23,24)$, two prospective studies were performed to identify the FNR of SLN mapping in this patient population. The GROINS V study, a multi-institutional observational study, described a FNR of 5.9\% and FNPV of 2.9\% (25). In GOG 173, which mirrored our current study in endometrial cancer, patients were enrolled prospectively and underwent SLN biopsy followed by inguinal lymphadenectomy. This allowed for a comprehensive evaluation of the accuracy of SLN biopsy and determination of the true FNR. Levenback et al. reported a sensitivity of $91.7 \%$ and FNPV of $2.0 \%$ in patients with tumors $<4 \mathrm{~cm}(26)$. Based on these data, SLN biopsy is now considered standard of care for select women with vulvar cancer.

The main strength of our study is the prospective evaluation of SLN mapping in women with high-risk endometrial cancer. Our study is the first prospective study to focus on high-risk patients with a higher overall risk of lymph node metastasis. All patients were required to have a comprehensive surgical staging which allowed us to accurately define the sensitivity, NPV and FNR. In addition, we chose a technique that was generalizable across practice types and is reproducible. Finally, the pathologic findings in our study mirrored the predicted outcomes for women with high-risk endometrial cancer. Our overall rate of positive lymph nodes was $23 \%$, consistent with the predicted risk of $25 \%$. Among patients with positive nodes, the SLN was the only positive node in $40 \%$ as described by other authors (11). Approximately $50 \%$ of patients that had positive pelvic nodes also had positive 
para-aortic nodes (27). There was one patient (1\%) with metastatic disease to an isolated para-aortic node.

The main limitation of the study is that it was performed at a single, large tertiary referral center. While 14 different surgeons participating in the study supports generalizability, the patient population and the resources at a cancer center may be different than a community setting. In addition, there were differences in the type of dye used based on the surgical approach. While some would argue that using the exact same technique is the only way to validate SLN mapping, the resources available at different centers also affects the type of dye available. In addition, there may be continued concern that a cervical injection which maps the lower half of the uterus, may not accurately map a fundal tumor or identify patients with an isolated para-aortic node. Our data combined with other published studies, however, show that cervical injection is not only accurate but reproducible across multiple practice settings. As more data become available and the technology improves, the technique to identify SLN in endometrial cancer should evolve. For example, Martinelli et al., recently described a technique of peri-tumoral injection of ICG which resulted in an overall SLN detection rate of $89.5 \%$. Interestingly, $47 \%$ mapped to the para-aortic nodes and $74.5 \%$ mapped to bilateral pelvic nodes (28).

At the completion of our study, we implemented a systematic change in how we stage patients with high-risk endometrial cancer. While comprehensive surgical staging was our previous standard, we have moved to SLN biopsy, hysterectomy, and bilateral salpingooophorectomy. In patients that do not map bilaterally, side-specific pelvic lymphadenectomy and para-aortic lymphadenectomy are performed. While our study addresses the accuracy of SLN biopsy in identifying patients with positive nodes, it does not address the use of adjuvant therapy in patients found to have a positive pelvic SLN. In a careful review of the node positive patients, $48 \%$ (11/23) also had positive para-aortic nodes. We found that uterine factors such as deep myometrial invasion could help identify which node-positive patients may benefit from extended field radiation therapy. We will continue to follow patients for compliance with this treatment strategy, as well as outcomes and overall survival for these patients.

In this prospective study on SLN mapping in women with high-risk endometrial cancer with comprehensive surgical staging, we confirmed a SLN detection rate of close to $90 \%$. SLN biopsy was able to accurately identify $95 \%$ of women with positive lymph nodes. With the implementation of a SLN algorithm, the FNR was $4.3 \%$ and FNPV was $1.4 \%$. These findings support SLN mapping in high-risk women and validate the algorithm of sidespecific lymphadenectomy if a SLN is not identified. Long-term studies will be needed to determine the impact of SLN biopsy alone on survival of women with high-risk endometrial cancer.

\section{Acknowledgments}

Funding: This work was supported in part by Cancer Center Support Grant (NCI Grant P30 CA016672), Andrew Sabin Family Fellowship, and NCI SPORE in Uterine Cancer (2P50 CA098258-06). 


\section{References}

1. Siegel RL, Miller KD, Jemal A. Cancer statistics, 2016. CA: a cancer journal for clinicians. 2016; 66(1):7-30. [PubMed: 26742998]

2. Benedetti Panici P, Basile S, Maneschi F, Alberto Lissoni A, Signorelli M, Scambia G, et al. Systematic pelvic lymphadenectomy vs. no lymphadenectomy in early-stage endometrial carcinoma: randomized clinical trial. Journal of the National Cancer Institute. 2008; 100(23):170716. [PubMed: 19033573]

3. Kitchener H, Swart AM, Qian Q, Amos C, Parmar MK. group As. Efficacy of systematic pelvic lymphadenectomy in endometrial cancer (MRC ASTEC trial): a randomised study. Lancet. 2009; 373(9658):125-36. [PubMed: 19070889]

4. DiSaia PJ, Creasman WT, Boronow RC, Blessing JA. Risk factors and recurrent patterns in Stage I endometrial cancer. American journal of obstetrics and gynecology. 1985; 151(8):1009-15. [PubMed: 3985062]

5. Barlin JN, Zhou Q, St Clair CM, Iasonos A, Soslow RA, Alektiar KM, et al. Classification and regression tree (CART) analysis of endometrial carcinoma: Seeing the forest for the trees. Gynecologic oncology. 2013; 130(3):452-6. [PubMed: 23774300]

6. Dowdy SC, Borah BJ, Bakkum-Gamez JN, Weaver AL, McGree ME, Haas LR, et al. Prospective assessment of survival, morbidity, and cost associated with lymphadenectomy in low-risk endometrial cancer. Gynecologic oncology. 2012; 127(1):5-10. [PubMed: 22771890]

7. Yost KJ, Cheville AL, Al-Hilli MM, Mariani A, Barrette BA, McGree ME, et al. Lymphedema after surgery for endometrial cancer: prevalence, risk factors, and quality of life. Obstetrics and gynecology. 2014; 124(2 Pt 1):307-15. [PubMed: 25004343]

8. Rossi EC, Jackson A, Ivanova A, Boggess JF. Detection of sentinel nodes for endometrial cancer with robotic assisted fluorescence imaging: cervical versus hysteroscopic injection. Int J Gynecol Cancer. 2013; 23(9):1704-11. [PubMed: 24177256]

9. Jewell EL, Huang JJ, Abu-Rustum NR, Gardner GJ, Brown CL, Sonoda Y, et al. Detection of sentinel lymph nodes in minimally invasive surgery using indocyanine green and near-infrared fluorescence imaging for uterine and cervical malignancies. Gynecologic oncology. 2014; 133(2): 274-7. [PubMed: 24582865]

10. Plante M, Touhami O, Trinh XB, Renaud MC, Sebastianelli A, Grondin K, et al. Sentinel node mapping with indocyanine green and endoscopic near-infrared fluorescence imaging in endometrial cancer. A pilot study and review of the literature. Gynecologic oncology. 2015; 137(3):443-7. [PubMed: 25771495]

11. Paley PJ, Veljovich DS, Press JZ, Isacson C, Pizer E, Shah C. A prospective investigation of fluorescence imaging to detect sentinel lymph nodes at robotic-assisted endometrial cancer staging. American journal of obstetrics and gynecology. 2016; 215(1):117e1-7. [PubMed: 26743505]

12. Holloway RW, Gupta S, Stavitzski NM, Zhu X, Takimoto EL, Gubbi A, et al. Sentinel lymph node mapping with staging lymphadenectomy for patients with endometrial cancer increases the detection of metastasis. Gynecologic oncology. 2016; 141(2):206-10. [PubMed: 26905211]

13. Ballester M, Dubernard G, Lecuru F, Heitz D, Mathevet P, Marret H, et al. Detection rate and diagnostic accuracy of sentinel-node biopsy in early stage endometrial cancer: a prospective multicentre study (SENTI-ENDO). The lancet oncology. 2011; 12(5):469-76. [PubMed: 21489874]

14. Barlin JN, Khoury-Collado F, Kim CH, Leitao MM Jr, Chi DS, Sonoda Y, et al. The importance of applying a sentinel lymph node mapping algorithm in endometrial cancer staging: beyond removal of blue nodes. Gynecologic oncology. 2012; 125(3):531-5. [PubMed: 22366409]

15. NCCN Clinical Practice Guidelines in Oncology. Uterine Neoplasms. Available from: http:// www.nccn.org/professionals/physician_gls/pdf/uterine.pdf

16. Thall PF, Simon RM, Estey EH. Bayesian sequential monitoring designs for single-arm clinical trials with multiple outcomes. Stat Med. 1995; 14(4):357-79. [PubMed: 7746977]

17. Harris PA, Taylor R, Thielke R, Payne J, Gonzalez N, Conde JG. Research electronic data capture (REDCap)--a metadata-driven methodology and workflow process for providing translational 
research informatics support. Journal of biomedical informatics. 2009; 42(2):377-81. [PubMed: 18929686]

18. Burke TW, Levenback C, Tornos C, Morris M, Wharton JT, Gershenson DM. Intraabdominal lymphatic mapping to direct selective pelvic and paraaortic lymphadenectomy in women with high-risk endometrial cancer: results of a pilot study. Gynecologic oncology. 1996; 62(2):169-73. [PubMed: 8751545]

19. Solima E, Martinelli F, Ditto A, Maccauro M, Carcangiu M, Mariani L, et al. Diagnostic accuracy of sentinel node in endometrial cancer by using hysteroscopic injection of radiolabeled tracer. Gynecologic oncology. 2012; 126(3):419-23. [PubMed: 22659192]

20. Frumovitz M, Bodurka DC, Broaddus RR, Coleman RL, Sood AK, Gershenson DM, et al. Lymphatic mapping and sentinel node biopsy in women with high-risk endometrial cancer. Gynecologic oncology. 2007; 104(1):100-3. [PubMed: 16963111]

21. Abu-Rustum NR, Khoury-Collado F, Gemignani ML. Techniques of sentinel lymph node identification for early-stage cervical and uterine cancer. Gynecologic oncology. 2008; 111(2 Suppl):S44-50. [PubMed: 18760450]

22. Smith AJ, Fader AN, Tanner EJ. Sentinel Lymph Node Assessment in Endometrial Cancer: A Systematic Review and Meta-analysis. American journal of obstetrics and gynecology. 2016

23. Rob L, Robova H, Pluta M, Strnad P, Kacirek J, Skapa P, et al. Further data on sentinel lymph node mapping in vulvar cancer by blue dye and radiocolloid Tc99. Int J Gynecol Cancer. 2007; 17(1): 147-53. [PubMed: 17291246]

24. Levenback C, Coleman RL, Burke TW, Bodurka-Bevers D, Wolf JK, Gershenson DM. Intraoperative lymphatic mapping and sentinel node identification with blue dye in patients with vulvar cancer. Gynecologic oncology. 2001; 83(2):276-81. [PubMed: 11606084]

25. Van der Zee AG, Oonk MH, De Hullu JA, Ansink AC, Vergote I, Verheijen RH, et al. Sentinel node dissection is safe in the treatment of early-stage vulvar cancer. J Clin Oncol. 2008; 26(6): 884-9. [PubMed: 18281661]

26. Levenback CF, Ali S, Coleman RL, Gold MA, Fowler JM, Judson PL, et al. Lymphatic mapping and sentinel lymph node biopsy in women with squamous cell carcinoma of the vulva: a gynecologic oncology group study. J Clin Oncol. 2012; 30(31):3786-91. [PubMed: 22753905]

27. Creasman WT, Morrow CP, Bundy BN, Homesley HD, Graham JE, Heller PB. Surgical pathologic spread patterns of endometrial cancer. A Gynecologic Oncology Group Study. Cancer. 1987; 60(8 Suppl):2035-41. [PubMed: 3652025]

28. Martinelli F, Ditto A, Bogani G, Signorelli M, Chiappa V, Lorusso D, et al. Laparoscopic Sentinel Node Mapping in Endometrial Cancer After Hysteroscopic Injection of Indocyanine Green. Journal of minimally invasive gynecology. 2016 


\section{Highlights}

- Sentinel lymph detection rates were $89 \%$ in high risk patients

- The sensitivity of SLN was $95 \%$ in high-risk endometrial cancer

- The FNR of SLN with side-specific LAD when an SLN is not detected is $4.3 \%$. 


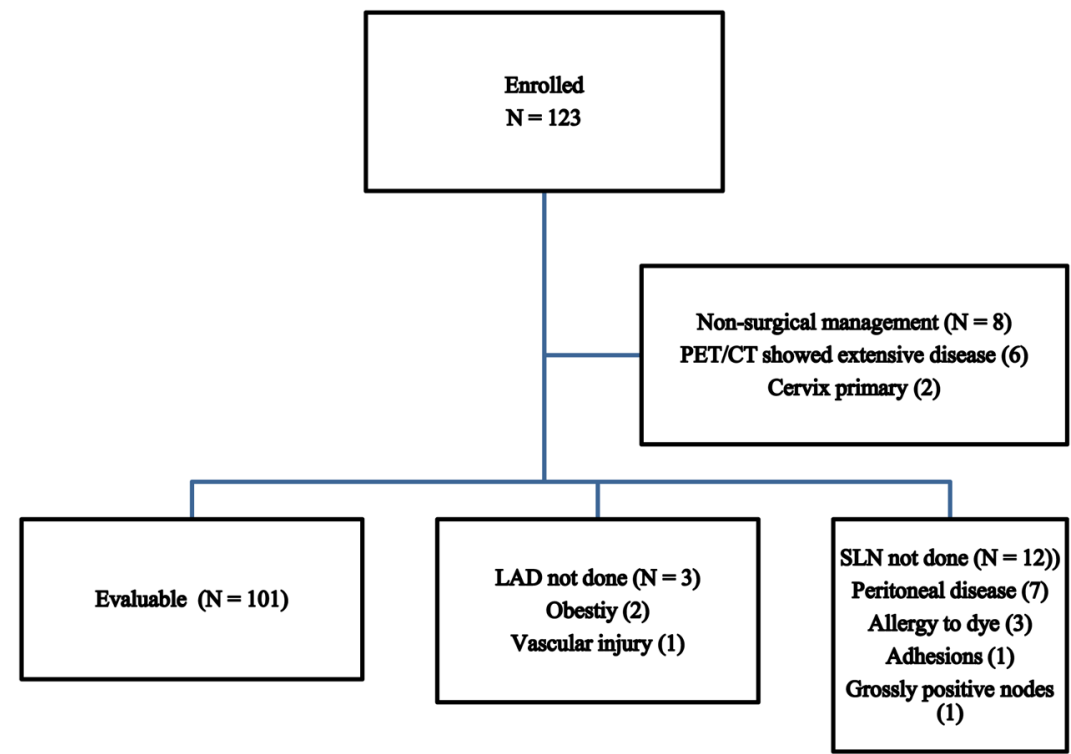

Figure 1.

Consort diagram of patient enrollment. 


\section{Table 1}

Sentinel lymph node dye used based on surgical approach

\begin{tabular}{|l|l|l|l|l|}
\hline Surgical approach & Dye & Concentration & Total Volume & Time of injection \\
\hline Robotic $*$ & Indocyanine green & $1.25 \mathrm{mg} / \mathrm{mL}$ & $4 \mathrm{cc}$ & After the abdomen is entered \\
\hline Laparoscopy & Blue dye (Lymphazurin) & $1 \%$ & $4 \mathrm{cc}$ & After the abdomen is entered \\
\hline & Technitium-99 & $0.5-1.0 \mathrm{mCi}$ & $2 \mathrm{cc}$ & $\begin{array}{l}\text { After the patient is asleep, prior to entering the } \\
\text { abdomen }\end{array}$ \\
\hline & Indocyanine green & $1.25 \mathrm{mg} / \mathrm{mL}$ & $4 \mathrm{cc}$ & After the abdomen is entered \\
\hline Laparotomy & Blue dye (Lymphazurin) & $1 \%$ & $4 \mathrm{cc}$ & After the abdomen is entered \\
\hline & Technitium-99 & $1.1 \mathrm{mCI}$ & $2 \mathrm{cc}$ & $\begin{array}{l}\text { After the patient is asleep, prior to entering the } \\
\text { abdomen }\end{array}$ \\
\hline
\end{tabular}

Firefly technology (da Vinci Surgical Systems, Sunnyvale, CA)

** Use of ICG became available in November, 2015 using Pinpoint technology (Novadaq Technologies', Inc., Mississauga, Ontario) 


\section{Table 2}

Demographic Characteristics

\begin{tabular}{|c|c|}
\hline & $N=101$ \\
\hline Median Age & 62 years $(29-86)$ \\
\hline Median BMI & $30.8 \mathrm{~kg} / \mathrm{m}^{2}(15.8-64.3)$ \\
\hline \multicolumn{2}{|l|}{ Histology } \\
\hline Endometrioid & $44(44 \%)$ \\
\hline Serous & $30(30 \%)$ \\
\hline Clear cell & $16(16 \%)$ \\
\hline MMMT & $10(10 \%)$ \\
\hline Other & 1 \\
\hline \multicolumn{2}{|l|}{ Surgical approach } \\
\hline Laparoscopy & $44(44 \%)$ \\
\hline Robotic & $37(37 \%)$ \\
\hline Laparotomy & $17(17 \%)$ \\
\hline Combined laparoscopy and robotic & $3(3 \%)$ \\
\hline \multicolumn{2}{|l|}{ FIGO Stage } \\
\hline IA & $46(46 \%)$ \\
\hline IB & $15(15 \%)$ \\
\hline II & $12(12 \%)$ \\
\hline IIIA & $4(4 \%)$ \\
\hline IIIC1 & $10(10 \%)$ \\
\hline IIIC2 & $10(10 \%)$ \\
\hline IV & $4(4 \%)$ \\
\hline
\end{tabular}




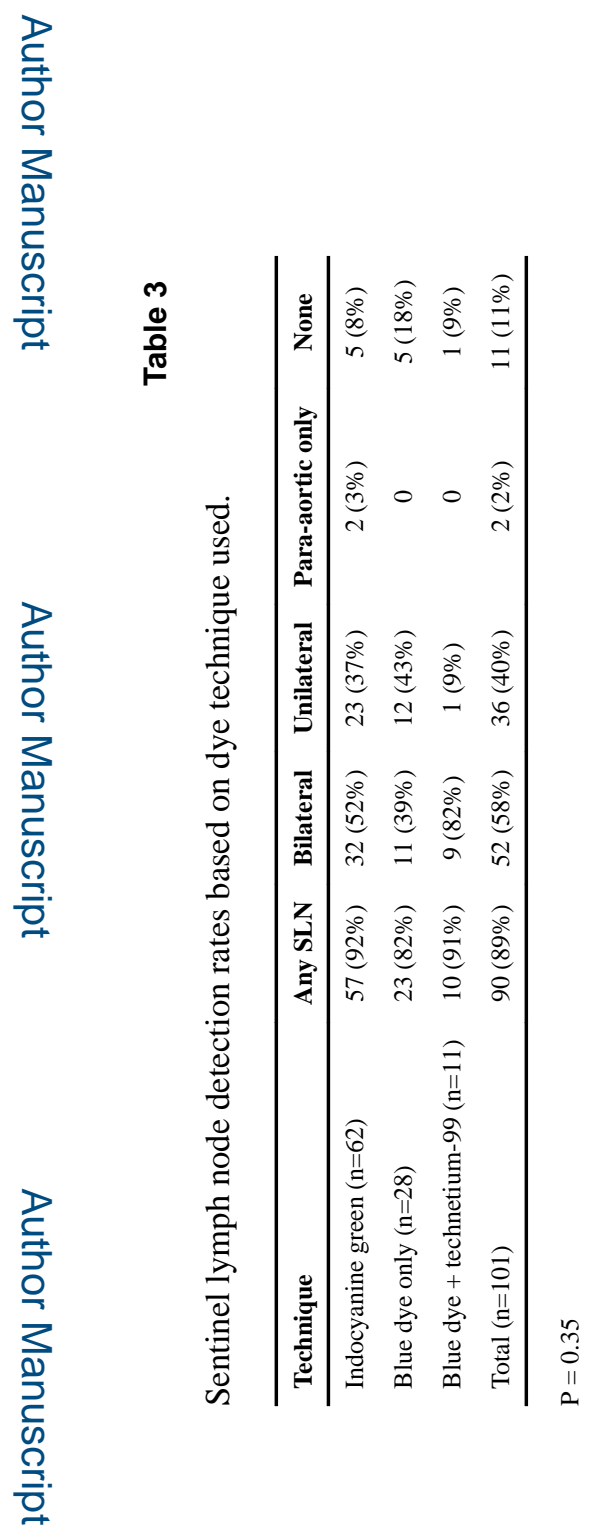


Table 4

Sentinel lymph node detection rates by hemi-pelvis (A) and patient (B).

\begin{tabular}{|c|c|c|c|c|}
\hline \multicolumn{4}{|c|}{$\begin{array}{l}\text { Table 4A. Detection Rates by Hemi-Pelvis } \\
\text { Lymph Nodes }\end{array}$} & \multirow[b]{2}{*}{ Tota } \\
\hline \multirow{4}{*}{ SLN } & \multirow{3}{*}{$\begin{array}{l}\text { Positive } \\
\text { Negative }\end{array}$} & Negative & Positive & \\
\hline & & 0 & 26 & 26 \\
\hline & & 98 & 2 & 100 \\
\hline & \multirow[t]{2}{*}{ Total } & 98 & 28 & 126 \\
\hline & & \multicolumn{2}{|c|}{$95 \%$ Confidence Interval } & \\
\hline Sensitivity & $92.9 \%$ & $76.5 \%$ & $99.1 \%$ & \\
\hline NPV & $98.0 \%$ & $93.0 \%$ & $99.8 \%$ & \\
\hline FNR & $7.1 \%$ & $0.9 \%$ & $23.5 \%$ & \\
\hline FNPV & $2 \%$ & $0.2 \%$ & $7.0 \%$ & \\
\hline
\end{tabular}

\begin{tabular}{|c|c|c|c|c|}
\hline \multicolumn{5}{|c|}{ Table 4B. Detection Rates by Patient } \\
\hline \multirow{4}{*}{ SLN } & \multirow[b]{3}{*}{ Positive } & \multicolumn{2}{|c|}{ Lymph Nodes } & \multirow[b]{2}{*}{ Total } \\
\hline & & Negative & Positive & \\
\hline & & 0 & 19 & 19 \\
\hline & Negative & 70 & 1 & 71 \\
\hline & Total & 70 & 20 & 90 \\
\hline & & $95 \%$ Confic & ce Interval & \\
\hline Sensitivity & $95.0 \%$ & $75.1 \%$ & $99.9 \%$ & \\
\hline NPV & $98.6 \%$ & $92.4 \%$ & $99.97 \%$ & \\
\hline FNR & $5 \%$ & $0.1 \%$ & $24.9 \%$ & \\
\hline FNPV & $1.4 \%$ & $0.03 \%$ & $7.6 \%$ & \\
\hline
\end{tabular}

